\title{
Robust Logistics Network Modeling and Design against Uncertainties*
}

\author{
Yoshiaki SHIMIZU**, Hideaki FUSHIMI** and Takeshi WADA*** \\ ** Mechanical Engineering, Toyohashi University of Technology \\ Tenpaku-cho, Toyohashi, Aichi, 441-8580, Japan \\ E-mail: shimizu@me.tut.ac.jp \\ *** Industrial Systems Engineering, Osaka Prefectural College of Technology \\ Saiwai-cho, Neyagawa, Osaka 572-8572, Japan
}

\begin{abstract}
In design of logistics networks, it is inevitable to consider various forward factors that will often change from their reference values. With this point of view, this study formulates a global logistics network design problem under uncertainties and present a solution method in terms of the stochastic programming known as recourse model. To reduce the effort to solve the resulting problem, a scenario-base approach is adopted instead of considering infinite realizations of uncertain parameters and sample average approximation. Furthermore, we present a method to support decision-making on robust design under noisy environment in terms of the knowledge from multi-objective optimization. Numerical experiments revealed validity of the proposed method through comparison with the reference results.
\end{abstract}

Key words : Logistics, Stochastic Programming, Recourse Model, Hybrid Tabu Search, Robust Design.

\section{Introduction}

Due to the recent global production and unstable business climate, it is becoming more and more difficult to predict the economic fabric of society. Eventually, this means companies are required to respond more adaptively and quickly for the customer demands and market changes so as to overcome the price war. Having a keen awareness of effective unification of supply chain and business alliance, we must aim at improvement of service and cost saving in manufacturing. As a core of such idea, it is of special importance to design, and manage logistics associated with various uncertainties involved in decision environment. In the design of logistics networks, it is inevitable to consider various forward factors such as customer demand, raw material price, currency exchanges, and so on. Since it is extremely difficult to forecast correctly these parameters at designing phase, a countermeasure against the uncertainties is to consider them at operation phase.

With this point of view, in this study, we present a solution method of the uncertain global logistics network design problem in terms of the stochastic programming known as recourse model. There, we adopted a scenario-base approach instead of considering infinite realizations of the uncertain parameters to depress computation load without losing the essence of the problem. Furthermore, we present a method to support decision-making on robust design under noisy environment by virtue of the knowledge from multi-objective optimization. Finally, we provide a few numerical experiments to validate effectiveness of the proposed method.

The aim of this study is to extend our generic idea (Shimizu \& Wada, 2004; Shimizu, Matsuda \& Wada, 2006) to cope with real world applications that are inseparable from the unstable and unpredictable circumstance. 


\section{Problem for Mulation}

\subsection{Related studies}

Recently, a review on facility location problem within the context of supply chain management (SCM) is taken place for the articles published in the last decade (Melo, Nickel \& Saldanha-da-Gama, 2009). While interests thereat are focused mainly on the strategic decision making and limited to discrete location problems, they revealed an increasing need for extensive studies and a still scarceness of models that capture simultaneously many aspects relevant to real-life applications. In fact, they showed most of the surveyed papers concerned rather simple deterministic single-period problems. Amazingly, only one paper features the stochastic multi-period problem. The followings are recent studies associated with the uncertainties.

Focusing on fluctuating demands, capacity expansion and reduction scenarios are analyzed by Melo, Nickel \& Saldanha-da-Gama (2005). A two-echelon logistics system design optimization is studied so that the probability of each customer to be covered is not less than a critical service level. Then, a vehicle routing problem is formulated using an improved genetic algorithm and a certain clustering technique based on traveling distance (Hwang, 2002). A transportation-inventory network design problem is studied under uncertain demand of retailers. They formulated the problem as a set-covering integer-programming and proposed to apply the column generation algorithm by exploiting certain special structures (Shu, Teo \& Shen, 2005). A simultaneous approach to incorporate inventory control decisions into typical facility location model is considered in the distribution network design problem. The problem is formulated as a non-linearmixed-integer programming considering a stochastic demand and a heuristic solution approach based on Lagrangian relaxation and the sub-gradient method is given (Miranda \& Garrido, 2004). A location-distribution-inventory model for a three-echelon supply chain is concerned under stochastic demands by Miranda and Garrido (2008). They developed a heuristic based on Lagrangian relaxation to find a lower bound of the objective function. A procedure based on $k$-opt-type local search is additionally applied to find a heuristic solution. A logistic model that combines facility location with spare part management is developed by Ommeren et al. (2006). Under exponential service time and Poissonian demand, they propose a local search heuristic for its solution. To deal with the higher degree of uncertainty in a reverse logistics context, MILP facility-location models are extended to $M I N L P$-model associated with queuing relationships. Then, an algorithm is proposed based on the technique of differential evolution to find robust solutions within an acceptable time limit (Lieckens \& Vandaele, 2007).

However, since the above all studies concerned only with small size problem, it seems hard to be applied in real-life problems. Apart from the uncertainty, we have solved various large scale logistics optimization problems under a variety of integrated real-life conditions. (Shimizu \& Wada, 2004; Wada, Shimizu \& Yoo, 2005; Wada, Yamazaki \& Shimizu, 2007; Shimizu, Yamazaki \& Wada, 2008). Within the same framework of these, we applied an idea of flexibility to cope with the demand uncertainty associated with the service level (Shimizu, Matsuda \& Wada, 2006).

The aim of this paper is to show the potential of our generic idea and to develop an extended method to cope with the uncertainties that are inseparable from unstable and unpredictable real-life circumstance.

\subsection{Deterministic Model}

First, let us describe a basic deterministic model by taking a hierarchical logistics network whose members are composed of plants, distribution centers (DCs), wholesaler, retailer and customers as shown in Fig.1.

$$
\text { (p.1) Min } f(x, y)=\sum_{k \in K} F_{k} y_{k}+\sum_{j \in J} \sum_{k \in K} B_{j} x_{3 j k}
$$




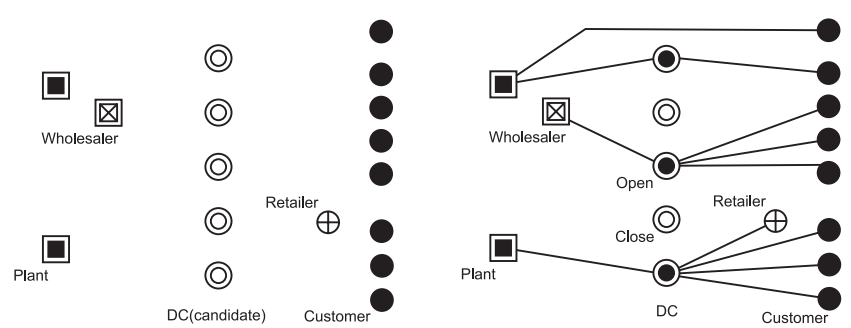

Fig. 1 Design problem of SCM under consideration (Members of SCM (left), Designed network of SCM (right)

$+\sum_{i \in I} \sum_{k \in K} C_{i} x_{1 i k}+\sum_{i \in I} \sum_{m \in M} C_{i} x_{2 i m}$

$+\sum_{k \in K} \sum_{l \in L} H_{k} x_{4_{k l}}+\sum_{k \in K} \sum_{m \in M} H_{k} x_{5_{k m}}$

$+\sum_{i \in I} \sum_{k \in K} E_{1_{i k}} x_{1_{i k}}+\sum_{i \in I} \sum_{m \in M}\left(E_{2_{i m}}-S_{m}\right) x_{2_{i m}}$

$+\sum_{j \in J} \sum_{k \in K} E_{3_{j k}} x_{3 j k}$

$+\sum_{k \in K} \sum_{l \in L}\left(E_{4_{k l}}-R_{l}\right) x_{4_{k l}}+\sum_{k \in K} \sum_{m \in M}\left(E_{5_{k m}}-S_{m}\right) x_{5_{k m}}$

subject to

$\sum_{i \in I} x_{2_{i m}}+\sum_{k \in K} x_{5_{k m}}=D_{m} \quad \forall m \in M$,

$\sum_{i \in I} x_{1_{i k}}+\sum_{j \in J} x_{3 j k} \leq U_{k} y_{k} \quad \forall k \in K$

$\sum_{i \in I} x_{1_{i k}}+\sum_{j \in J} x_{3_{j k}}=\sum_{l \in L} x_{4_{k l}}+\sum_{m \in M} x_{5_{k m}} \quad \forall k \in K$

$\sum_{k \in K} x_{1_{i k}}+\sum_{m \in M} x_{2_{i m}} \leq P_{i}^{\max } \quad \forall i \in I$

$\sum_{k \in K} x_{1_{i k}}+\sum_{m \in M} x_{2_{i m}} \geq P_{i}^{\min } \quad \forall i \in I$

$x=\left(x_{1} \oplus x_{2} \oplus x_{3} \oplus x_{4} \oplus x_{5}\right) \in \mathbb{R}, y \in\{0,1\}$

where notation $\oplus$ means the direct sum of vector and the others denote as follows:
$B_{j} \quad$ : unit purchase price from wholesaler $j$
$C_{i} \quad$ : unit production cost at plant $i$
$D_{m} \quad$ : demand of customer $m$
$E_{r_{i j}} \quad$ : unit transport cost between $i$ and $j$
belonging to member $r,(r=1, \ldots, 5)$
$x_{r_{i j}} \quad:$ shipped amount from $i$ to $j$
belonging to member $r,(r=1, \ldots, 5)$
$F_{k} \quad$ : fixed-charge cost for opening DC $k$
$H_{k} \quad$ : unit handling cost at DC $k$
$P_{i}^{\max } \quad$ : upper bound of production capacity at plant $i$
$P_{i}^{\min } \quad$ : lower bound of production capacity at plant $i$
$R_{l} \quad$ : unit selling price to retailer $l$
$S_{m} \quad$ : unit selling price to customer $m$
$U_{k} \quad$ : upper bound of holding capacity at DC $k$
$y_{j} \quad$ : take 1 if DC $j$ is open, otherwise 0
$I, J, K, \quad$ : index set describing plant, wholesaler, DC,
$L, M \quad$ retailer and customer, respectively 
Moreover, regarding the member, $r=1$ means the path from plant to DC (plant-DC, in short) and similarly as follows 2:plant-customer; 3:wholesaler-DC; 4:DC-retailer; 5:DCcustomer.

The objective function is the total cost composed of fixed-charge for opening DCs (first), purchase costs from wholesalers (2nd), production costs at plants ( $3 \mathrm{rd}$ and 4 th), handling costs at DCs (5th and 6th), and transportation costs (Thru the 7th to the 11th, sales for customers are subtracted in the 8 th and the 11th and sales for retailers in the 10th), respectively.

On the other hand, we impose the constraints on demand satisfaction of every customer Eq.(1), holding capacity bound at each DC, Eq.(2), input-output balance at each DC Eq.(3), available amount of product at each plant, Eqs.(4) and (5). In addition, binary decision variables $y$ are introduced for selection of open/close of DCs while non-negative real variables $x$ for transport amounts.

\subsection{Stochastic Model}

In global and strategic planning of logistics, it is indispensable to take the following uncertain parameters involved in the foregoing deterministic model into account. They are unit purchase price from wholesaler $j, B_{j}(\forall j)$; unit production cost at plant $i, C_{i}(\forall i)$; demand of customer $m, D_{m}(\forall m)$; transportation cost between members per unit amount, $E_{r_{i j}}(\forall r, i, j)$; fixed-charge cost for opening DC $k, F_{k}(\forall k)$; unit handling cost at DC $k, H_{k}(\forall k)$; unit selling price to retailer $l, R_{l}(\forall l)$; and unit selling price to customer $m, S_{m}(\forall m)$.

In addition, it is rational to distinguish the decision variables into two classes, i.e., hard one that is impossible to change once decided even if the situation would change and the other (soft) possible to change according to the changed situation. Presently, we notice the decision on the location of DCs $\left(y_{k}, \forall k\right)$ correspond to the hard one while the transportation amounts $\left(x_{r_{i j}}, \forall r, i, j\right)$ to the soft one. Moreover, we can separate the terms of objective function into two classes, i.e., the fixed-charge cost which is a function of the hard variables and the other operating costs a function of the soft variables. The former is viewed as a goal for the decision made now while the later ones according to the consequence in future. Then, we can naturally take the stochastic programming model known as recourse model. It is described generally as follows (Kall \& Wallace, 1994; Gunderson, Morris \& Thompson, 1978).

$$
\text { Min } f_{1}(y)+\mathbb{E}\left[f_{2}(x(\xi), \xi)\right] \quad \text { subject to }\left\{\begin{array}{l}
g_{i}(y) \leq 0,(i=1, \ldots, m) \\
h_{j}(y, x(\xi)) \leq 0,(j=1, \ldots, k) \\
\xi \in \Xi
\end{array}\right.
$$

In this paradigm, $y$ is a vector of decisions that we must take now, and $x(\xi)$ is a vector of decision chosen for each possible realization of uncertain parameters $\xi \in \Xi$ in future. Moreover, $\mathbb{E}[\cdot]$ denotes the expectation of the value in brackets. This recourse model is commonly extended into such a two-stage model that mainly directs to $y$ in the upper level while $x$ in the lower level. According to Santoso et al. (2005), a formulation for logistic problem is given as follows.

$$
\text { (p.2) } \underset{y}{\operatorname{Min}} f(y)=F^{\mathrm{T}} y+\mathbb{E}[Q(y, \xi)] \quad \text { subject to } \quad y \in Y, \quad \xi \in \Xi
$$

where $y$ denotes a binary vector that decides the DC status (open/close).

The first and second terms of the objective function are the fixed-charge and the expected value of the operating cost, respectively. Here, let's note the decision variables are only $y$ in the upper level problem.

The operating cost itself becomes the objective function at the lower level. In practice, $Q(y, \xi)$ denotes the optimal value of the following problem.

$\operatorname{Min}_{x, z} q^{\mathrm{T}} x+h^{\mathrm{T}} z \quad$ subject to 


$$
\begin{aligned}
& A x+B z \geq D \\
& G x \leq U y \\
& T x=0 \\
& W x \leq P \\
& x, z \in \mathbb{R}
\end{aligned}
$$

where $x\left(\Leftrightarrow{ }^{* 1} x_{r}, r=\{1,2,5\}\right)$ and $z\left(\Leftrightarrow x_{r}, r=\{3,4\}\right)$ are flow amounts between the members in the deterministic model.

The second term of the objective function $h^{T} z$ corresponds to the adjustment cost incurred to meet demand satisfaction. This can be interpreted as the outsourcing cost for unsatisfied demand from wholesaler and sales cost for retailer in the case of shortage and surplus production, respectively.

On the other hand, each constraint stands for customer demand requirement (Eq.(6) $\Leftrightarrow$ Eq.(1)), capacity constraint at DCs (Eq.(7) $\Leftrightarrow$ Eq.(2)), the material balance regarding the flow amounts (Eq.(8) $\Leftrightarrow$ Eq.(3)) and upper bound of production at plants (Eq.(9) $\Leftrightarrow$ Eqs.(4)/(5)), respectively.

In this two-stage stochastic model, the first-stage decides the configuration $y$ while the second-stage $x$ and $z$ processing and transporting products from plants to customers in an optimal fashion based upon the configuration and the realized uncertain parameters. The objective is to minimize current investment costs $F^{T} y$ and expected future operating costs $\mathbb{E}[Q(y, \xi)]$.

Under mild conditions, we know $Q(y, \xi)$ is finite for all $y \in Y$ and possible realization of the random parameters $\forall \xi$ in $\Xi$. Hence, the expected value $\mathbb{E}[Q(y, \xi)]$ is well defined and finite for the considered distribution of $\xi$. Consequently, upper level problem has a well-defined objective function and, possesses an optimal solution.

\section{Scenario-based Solution Method}

\subsection{Hybrid Approach}

As mentioned above, we adopted the formulation in the stochastic programming problem with recourse model. Since the basic idea of HybTS (Shimizu \& Wada,2004) is also similar two-level method as depicted in the left hand half of Fig.2, we can straightforwardly apply it for the present solution if we ignore the consideration on uncertainties at the moment. That is, in the solution procedure of HybTS, the upper level sub-problem decides the locations while the lower level sub-problem the network routes under the location prescribed at the upper level.

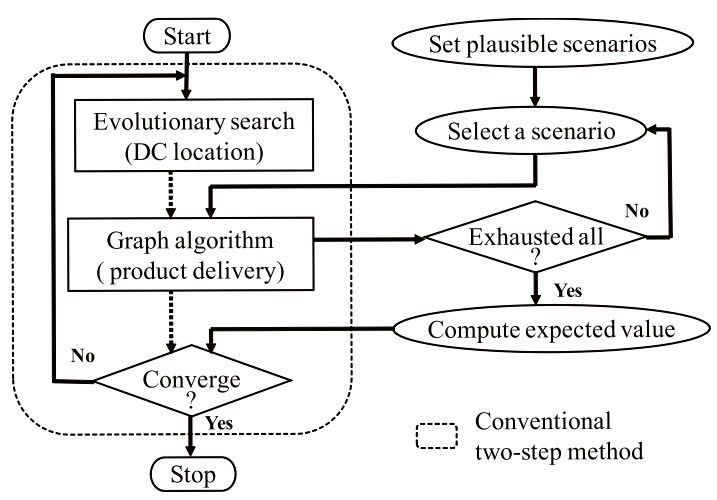

Fig. 2 Flow chart of the proposed algorithm

*1 Double-sided arrow $\Leftrightarrow$ denotes the correspondence to the deterministic model. 
Back to the uncertain case, however, we will notice the difficulty to practically evaluate the expected value associated with infinite realization of uncertain parameters. To overcome this problem, sample average approximation is reported (Santoso et al., 2005; Veraeij et al, 2003). This approach first transforms the original objective function as follows.

$$
f^{\prime}(y)=F^{\mathrm{T}} y+\frac{1}{N} \sum_{n=1}^{N} Q\left(y, \xi^{n}\right)
$$

where $\xi^{n}$ denotes the parameter realization for the $n$-th sample, $N$ denotes the size of sample. Then evaluating the optimality gap based on the average and variance values over the appropriately chosen samples, the final decision will be made. Mathematically, this approach presents a meaningful approximation method that makes it possible to reach the optimal ever. However, computational effort expands rapidly along with the increase in number of uncertain parameters. Consequently, serious problems are left behind for real-world applications.

We can resolve this problem practically if we notice the fact that independent deviation of every uncertain parameter would seldom occur. Generally speaking, uncertain parameters of logistic problems have somewhat correlations mutually, and their realization can be categorized into finite patterns given by the rate of variation to the nominal value. For the conceptional design, therefore, it is sufficient as well as efficient to adopt a scenario-based approach mentioned below.

For this purpose, we need to set up a typical set of scenarios regarding realization of uncertain parameters and its occurrence probability. Then the computation of expected value is given by:

$$
\mathbb{E}[Q(y, \xi)]=\sum_{n=1}^{N} p_{n} Q\left(y, \xi^{n}\right)
$$

where $\xi^{n}$ is a parameter realization of the $n$-th scenario, and $p_{n}$ its occurrence probability. Presently, $\xi^{n}$ is composed of the parameters mention in Section 2.3 and described as follows.

$$
\xi^{n}=\left(C_{i}^{n}, B_{j}^{n}, H_{k}^{n}, R_{l}^{n}, D_{m}^{n}, S_{m}^{n}, E_{r_{i j}}^{n}\right)
$$

After all, we have the algorithm outlined below (See also Fig.2).

Step 1: Decide the initial location of DC following the algorithm mentioned in the next section and go to Step 3.

Step 2: Update the location of DC by the sophisticated tabu search developed previously (Shimizu \& Wada, 2004).

Step 3: Solve the distribution problem for every scenario one after another at the lower-level under the prescribed DC location.

Step 4: From the above results, calculate the expected value by Eq.(11).

Step 5: If the stopping condition is satisfied, stop. Otherwise, evaluate the tentative solution and go back to Step 2.

In Step 3, since the lower level problem has a block diagonal structure as shown in Fig.3, we can independently solve the problem per each scenario. Moreover, each problem refers to a minimum cost flow (MCF) problem whose flow diagram is described as shown in Fig.4. Following the similar approach as before (Shimizu and Wada, 2004), we can obtain this graph by adding appropriate virtual nodes and edges so that the bounding constraints such as Eqs.(2), (4) and (5) are satisfied. To manage the adjusting term associated with variable $z$ in Eq.(6), the total amount of flow inlet or equivalently to say outlet flow is set as follows.

$$
\max \left\{\sum_{i} P_{i}^{\max }, \sum_{m} D_{m}\right\}
$$




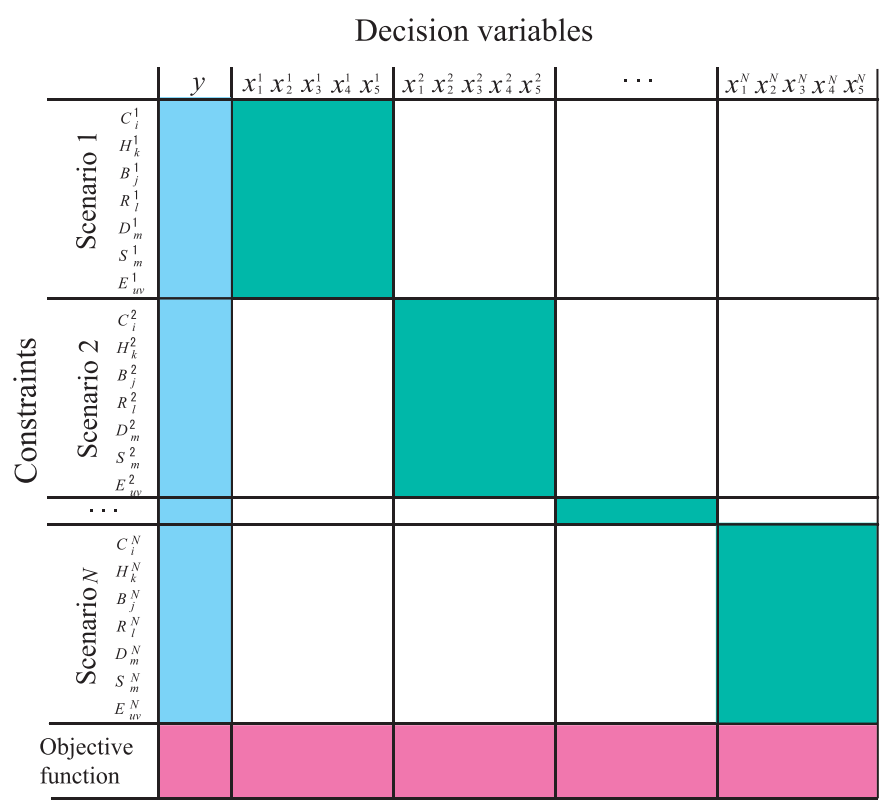

Fig. 3 Block diagonal structure of the integrated problem

\subsection{Special idea to decide the initial location}

As a common nature of meta-heuristic method, selection of the initial solution (location, presently) will greatly affect the performance of total efficiency of the algorithm. To work with this, in terms of the specific experience, we take the following procedure instead of the usual random selection. The procedure can provide a probable initial upper bound number of opening DC sites.

Step 1: Open all DC sites.

Step 2: Set some uncertain parameters as follows.

(1) Replace the unit handling cost of each scenario with the cheapest among all scenarios. $H_{k}:=\min \left\{H_{k}^{n} \mid n \in N\right\}, \quad \forall k \in K$

(2) Replace the demand of each scenario with the largest among all scenarios. $D_{m}:=\max \left\{D_{m}^{n} \mid n \in N\right\}, \quad \forall m \in M$

(3) Replace the transportation cost of each scenario with the cheapest one among all scenarios.

$E_{r_{i j}}:=\min \left\{E_{r_{i j}}^{n} \mid n \in N\right\}, \quad \forall r, i, j$

(4) Use the average values for the other uncertain parameters.

Step 3: By using the parameters decided at Step 2, solve the lower level problem, and select the DCs that are active, i.e., $x_{r_{i j}}>0(r=1,3)$ as members of the initial location.

\section{Robust Design}

In the above formulation, we know the occurrence probabilities will affect considerably the result. Even if we make great efforts for setting these values, they fluctuate somewhat in practice. It is desirable, therefore, to derive a robust solution that is less affected by the realization of these values. To cope with this problem, we propose a plain multi-objective approach mentioned below.

First, calculate the weighted average expected operating cost over the scenarios.

$Q_{a v}(y)=\sum_{n=1}^{N} p_{n} Q\left(y, \xi^{n}\right)$

Then, we obtain the variance as

$$
v(y)=\sum_{n=1}^{N} p_{n}\left(Q\left(y, \xi^{n}\right)-Q_{a v}(y)\right)^{2} .
$$




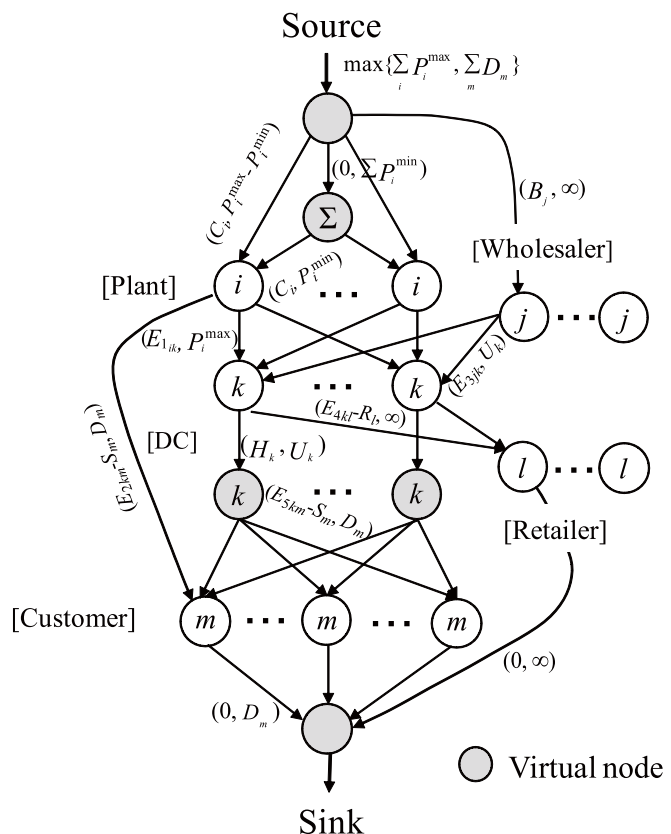

Fig. 4 Minimum cost flow graph for the present problem (Ignored some crowded edges and nodes for simplicity)

Eventually, we modify the objective function by the weighted sum of the original term and the new variance term as follow.

$$
\tilde{f}(y)=\alpha\left(F^{\mathrm{T}} y+Q_{a v}(y)\right)+(1-\alpha) v(y)
$$

where $\alpha$ denotes the weighting coefficient. Using this objective function instead of Eq.(10), we can derive the robust solution corresponding to the value of weight. That is, along with the decrease in $\alpha$, we will weigh the robustness more.

\section{Numerical Experiment and Discussions}

\subsection{Preliminary Result}

To verify the effectiveness of the proposed approach, we provided a few benchmark problems whose problem sizes range within the numbers shown in Table 1. System parameters are given randomly within their respective upper and lower bounded values. Table 2 shows the direction and the amounts of parameter deviations from the nominal values assumed for scenario D-M(b). Using the PC whose specification is shown in Table 3, we carried out the following numerical experiments.

From some preliminary experiments, we confirmed that the optimal DC location of each scenario is different from the others. This implicitly supports a significance to make a final decision while considering every realization of the scenario.

Table 1 Problem size of benchmark problem

\begin{tabular}{lcccccc}
\hline Data ID & $|I|$ & $|J|$ & $|K|$ & $|L|$ & $|M|$ & $N$ \\
\hline D-S & 3 & 1 & 30 & 1 & 50 & 3 \\
D-M (a/b) & 5 & 3 & 50 & 3 & 100 & 5 \\
D-L & 10 & 5 & 70 & 5 & 200 & 8 \\
\hline
\end{tabular}

\subsection{Comparison with the reference scenario}

We compared the proposed approach with the reference solution that is deterministically obtained using the parameters that are calculated as the weighed sum average using the occurrence probabilities, i.e., $\xi^{R}=\Sigma_{n} p_{n} \xi^{n}$. 
Table 2 Parameter realization for scenario D-M(b)

\begin{tabular}{ccccccccc}
\hline$n$ & $p_{n}$ & $C_{i}^{n}$ & $B_{j}^{n}$ & $H_{k}^{n}$ & $R_{l}^{n}$ & $D_{m}^{n}$ & $S_{m}^{n}$ & $E_{u v}^{n}$ \\
\hline 1 & $30 \%$ & $-10 \%$ & $+20 \%$ & $-10 \%$ & $+20 \%$ & $+20 \%$ & $+20 \%$ & $+20 \%$ \\
2 & $10 \%$ & $-10 \%$ & $-10 \%$ & $-10 \%$ & $-10 \%$ & $+20 \%$ & $-10 \%$ & $-10 \%$ \\
3 & $20 \%$ & $0 \%$ & $0 \%$ & $0 \%$ & $0 \%$ & $0 \%$ & $0 \%$ & $0 \%$ \\
4 & $25 \%$ & $+10 \%$ & $+20 \%$ & $+10 \%$ & $+20 \%$ & $-20 \%$ & $+20 \%$ & $+20 \%$ \\
5 & $15 \%$ & $+10 \%$ & $-10 \%$ & $+10 \%$ & $-10 \%$ & $-20 \%$ & $-10 \%$ & $-10 \%$ \\
\hline
\end{tabular}

Table 3 Computation environment

OS Debian GNU/Linux 3.1

Compiler The GNU C++ compiler. 2.95.4

CPU Intel ${ }^{\circledR}$ Pentium $^{\circledR} \quad 4 \quad 3.0[\mathrm{GHz}]$

Memory $512[\mathrm{MB}]$

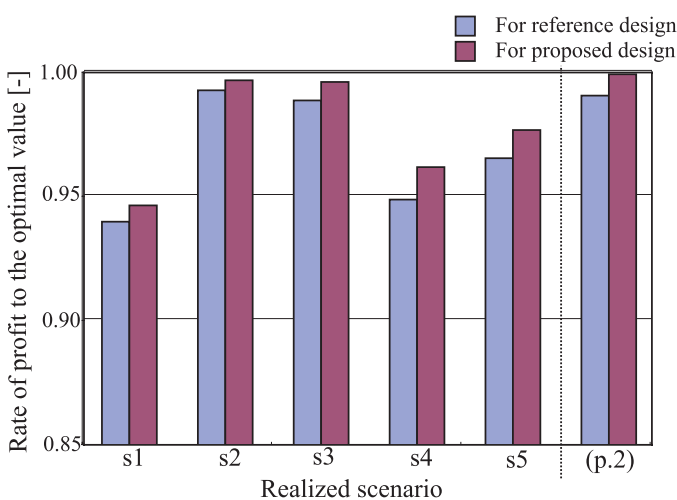

Fig. 5 Comparison of achievement rates per scenario when $N=5$ (Solved (p.2) in terms of Eq.(11) and Table 2)

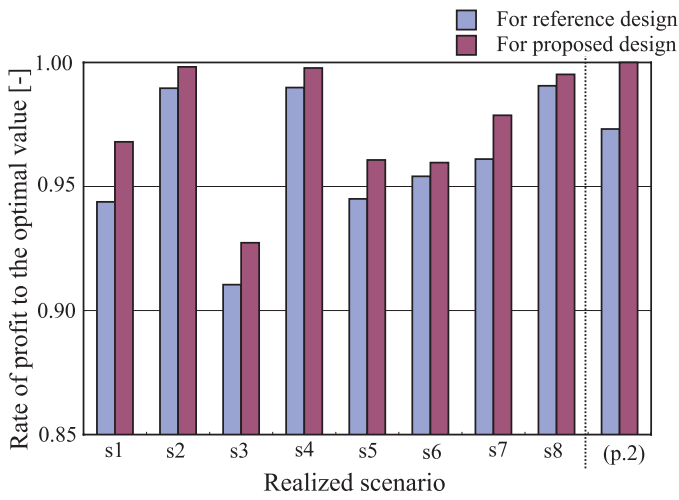

Fig. 6 Comparison of achievement rates per scenario when $N=8$ (Probability of every scenario is set at the same value) 
In Figs.5 \& 6, we compare the results when $N=5$ and $N=8$, respectively. From the achievement rates shown at (p.2) bars, we can see the advantage of the recourse over the prescribed approach. Furthermore, we reveal the favor from comparing the results when each decision is applied to the case where the respective object scenario (from s1 to s5) has occurred certainly or with probability 1 . There, a merit is calculated as an achievement rate to the optimal value under each scenario. Regardless of the scenario, the proposed approach outperforms the reference solution. Moreover, due to using the predetermined probability, the reference solution may not necessarily become feasible for every scenario. In contrast, the proposed solution can be always feasible since it must be feasible solution all over the scenarios (Refer to Figs. 2 and 3).

According to the increase in scenario number, this is equivalently to say, increase in uncertainty, effect of the proposed method became more clearly through comparison of these two figures since the differences of bar heights in Fig. 6 become larger than those in Fig.5.

\subsection{Enhancement of Solution Speed}

As supposed easily, according to the increase in number of scenarios, computing load expands rapidly. The selection of initial location also affect the convergence speed. To conserve the computation time, we invented a selection method of the initial location and changed the solution strategies in the course of search. In the earlier half, the reference (small probability weighed average) model is used to rapidly derive a near optimal solution. Then, we switched this reference model to the original distributed large model (See Fig.3) for the marginal search. By this strategy summarized in Fig.7, we can reduce the computation load significantly as shown in Table 4.

Table 4 Comparison of CPU time

\begin{tabular}{cccc}
\hline$N$ & Standard[sec] & Proposed[sec] & Reduced rate[\%] \\
\hline 3 & 36.51 & 22.26 & 38.9 \\
5 & 56.29 & 32.70 & 41.9 \\
8 & 93.63 & 50.03 & 46.6 \\
\hline
\end{tabular}

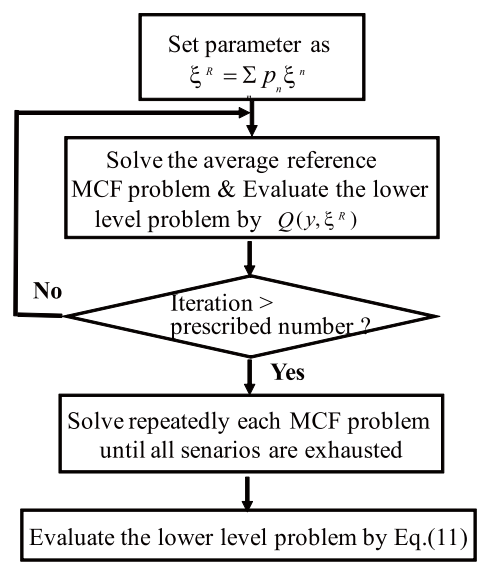

Fig. 7 Procedure for saving computation load

\subsection{Robust Design}

By solving repeatedly the problem under the objective function given by Eq.(15) with a set of $\alpha$, we derive a trade-off relation between the expected profit and the robustness or variance as shown in Fig.8. As supposed naturally, this trade-off curve locates between the maximum and minimum profit curves, and the robust strategy can be accomplished at the expense of the expected profit. It is very helpful for DM to decide his/her final decision referring to the observation of such profile of the trade-off curve since the profit degrades 


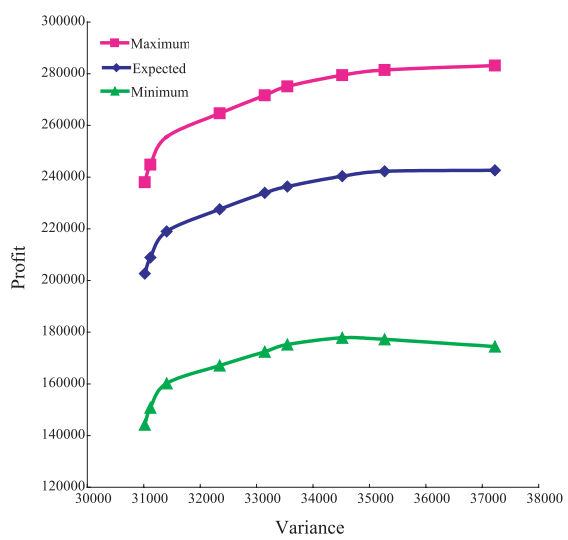

Fig. 8 Profiles of profits for the degree of robustness

greatly at the left edge of the curve.

\section{Conclusions}

In the design of logistics networks, it is inevitable to consider various forward factors that will often change from their reference values. Hence, we concerned with a global logistics network design problem under uncertainties. Noticing the similarity of problem structure between the recourse model in the stochastic programming and the conventional hierarchical solution method developed by the authors, we have developed a new two-stage stochastic model that is effective for the global logistics optimization. To reduce the effort to solve the problem, we adopted a scenario-base approach and proposed a method to support decision-making on robust design under noisy environment. We validated the rationale of our approach through numerical experiments.

Future studies should be devoted to improve the scenario-base method toward supporting the decision suitable at tactical level.

\section{References}

( 1 ) Bertsimas, D. and M. Sim. Robust discrete optimization and network flows. Math. Program, Ser. B, Vol. 98, pp. 49-71, 2003.

( 2 ) Gunderson, H. S., J. G. Morris and H. E. Thompson. Stochastic Programming with Recourse: A Modification from an Applications Viewpoint. Journal of the Operational Research Society, Vol. 29, pp. 769-778, 1978.

( 3 ) Hwang, H-S. Design of supply-chain logistics system considering service level. Computers $\mathcal{E}$ Industrial Engineering, Vol. 43, pp. 283-297, 2002.

( 4 ) Kall, P. and S. W. Wallace. Stochastic Programming. Wiley, Chichester. 1994.

( 5 ) Lieckens, K. and N. Vandaele. Reverse logistics network design with stochastic lead times. Computers $\mathcal{E}$ Operations Research, Vol. 34, pp. 395-416, 2007.

( 6 ) Melo M. T., S. Nickel and F. Saldanha-da-Gama. Dynamic multi-commodity capacitated facility location: A mathematical modeling framework for strategic supply chain planning. Computers $\mathcal{E}$ Operations Research, Vol. 33, pp. 181-208, 2005.

( 7 ) Melo, M. T., S. Nickel and F. Saldanha-da-Gama. Facility location and supply chain management: A review. European Journal of Operational Research, Vol. 196, pp. 401412, 2009

( 8 ) Miranda, P. A. and R. A. Garrido. Incorporating inventory control decisions into a strategic distribution network design model with stochastic demand. Transportation Research, Part E, Vol. 40, pp. 183-207, 2004.

( 9 ) Miranda, P. A. and R. A. Garrido. Valid inequalities for Lagrangian relaxation in an inventory location problem with stochastic capacity. Transportation Research, Part E, Vol. 44, pp. 47-65, 2008. 
(10) Ommeren, V. J. C. W., A. F. Bumb and A. V. Sleptchenko. Locating repair shops in a stochastic environment. Computers $\mathcal{E}$ Operations Research, Vol. 33, pp. 1575-1594, 2006.

(11) Santoso, T., S. Ahmed, M. Goetschalckx and A.Shapiro. A stochastic programming approach for supply chain network design under uncertainty. European Journal of Operational Research, Vol. 167, pp. 96-115, 2005.

(12) Shimizu, Y. and T. Wada. Logistic Optimization for Site Location and Route Selection under Capacity Constraints Using Hybrid Tabu Search. Proc. 8th Int. Symposium on Computer-Aided Process Systems Engineering, pp. 612-617, Kunming, China. 2004.

(13) Shimizu, Y., S. Matsuda, and T. Wada. A Flexible Design of Logistic Network against Uncertain Demands through Hybrid Meta-Heuristic Method. Proc. 16th Europe. Symposium on Computer-Aided Process Engineering, pp. 2051-2056, Garmisch Partenkirchen, Germany. 2006.

(14) Shimizu Y., Y. Yamazaki, and T. Wada. Multi-modal Logistics Network Design over Planning Horizon through a Hybrid Meta-heuristic Approach. Journal of Advanced Mechanical Design, Systems and Manufacturing, Vol. 25, pp. 915-925, 2008.

(15) Shimizu Y. and H. Kawamoto. An Implementation of Parallel Computing for Hierarchical Logistic Network Design Optimization Using PSO. 18th European Symposium on Computer Aided Process Engineering, pp. 605-610, Lyon, France, 2008.

(16) Shu, J., C-P. Teo and Z-J. M. Shen. Stochastic Transportation-Inventory Network Design Problem. Operations Research, Vol. 53, pp. 48-60, 2005.

(17) Verweji, B., S. Ahmed, A. J. Kleywegt, G. Nemheauser, and A. Shaprio. The Sample Average Approximation Method Applied to Stochastic Routing Problem, A Computational Study. Computational Optimization and Applications archive, Vol. 24, pp. 289333,2003

(18) Wada, T., Y. Shimizu, and J.-K. Yoo. Entire Supply Chain Optimization in Terms of Hybrid in Approach. Proc. 15th Europe. Symposium on Computer-Aided Process Engineering, pp. 1591-1596, Barcelona, Spain. 2005.

(19) Wada, T., Y. Yamazaki, and Y. Shimizu. Logistic Optimization Using Hybrid Metaheuristic Approach. Transaction of the Japan Society of Mechanical Engineers, C-727, Vol. 73, pp. 919-926, 2007. 